\title{
pGALS performs well in the hands of a medical student
}

\section{AL Rowan*1, S Jandial1', A Myers ${ }^{3}$, B Bateman ${ }^{3}$, M Friswell ${ }^{2}$ and HE Foster ${ }^{1}$}

Address: ${ }^{1}$ Newcastle University, Newcastle upon Tyne, UK, ${ }^{2}$ Newcastle Hospitals NHS Trust, Newcastle upon Tyne, UK and ${ }^{3}$ Northumbria Healthcare NHS trust, Northumberland, UK

* Corresponding author

from $15^{\text {th }}$ Paediatric Rheumatology European Society (PreS) Congress

London, UK. 14-17 September 2008

Published: 15 September 2008

Pediatric Rheumatology 2008, 6(SuppI I):PI52 doi:I0.II86/I546-0096-6-SI-PI52

This abstract is available from: http://www.ped-rheum.com/content/6/SI/PI52

(c) 2008 Rowan et al; licensee BioMed Central Ltd.

\section{Background}

pGALS (paediatric Gait, Arms, Legs and Spine) is a paediatric musculoskeletal (pMSK) screening examination validated for use in school-aged children [1], aimed at medical students. It is envisaged that pGALS will improve clinical skills and facilitate access to specialist care. Our aim was to assess the validity of pGALS in student hands and compare it to assessment by a consultant paediatric rheumatologist.

\section{Methods}

The student (AR) received standard undergraduate pMSK teaching. pGALS was performed on children attending paediatric rheumatology clinics, with the student blinded to diagnosis and background information. Findings were recorded as "abnormal" or "normal" and compared to a same day examination by a consultant (HF/MF).

\section{Results}

The study included 59 children, median age 12 (range 417 yrs). 45 (76\%) had juvenile idiopathic arthritis. Overall, sensitivity for whether a child was deemed "normal" or "abnormal" was $95 \%$, with specificity $88 \%$. Student pGALS had good sensitivity $(60-100 \%)$ and specificity (89-100\%) at all joints, except for TMJ (sensitivity 0\%, specificity 98\%). Missed abnormalities were mostly loss of range-of-movement at the foot, ankle and TMJ; however in these children, abnormalities elsewhere were detected. Student median time was 4.25 min (range 2.258.5), compared to consultant median of 2 min (range 2$8)[p=0.001]$. Pain score median was 0 (range $0-8$ ).

\section{Conclusion}

pGALS is quick, acceptable and performs well in determining if the child has a normal MSK examination or not when used by a student. However, interpretation of abnormal versus normal, especially at the foot, ankle and TMJ, need to be addressed in clinical teaching.

\section{References}

I. Foster HE, Kay LJ, Friswell M, Coady D, Myers A: pGALS - a paediatric musculoskeletal screening examination for school aged children based on the adult GALS screen. Arthritis Care Res 2006, 55:709-7I6. 